\title{
Single application hybrid interstitial brachytherapy for cervical cancer: an institutional approach during the COVID-19 pandemic
}

\author{
Shari Damast, MD', Christopher J. Tien, PhD!, Melissa Young, MD, PhD', Gary Altwerger, MD², Elena Ratner, MD² \\ 'Department of Therapeutic Radiology, Yale University School of Medicine, New Haven, CT, USA, ²Department of Obstetrics and Gynecology, \\ Yale University School of Medicine, New Haven, CT, USA
}

\begin{abstract}
Purpose: To ease anesthesia and inpatient strain during the COVID-19 pandemic, our institution's policy for hybrid intracavitary-interstitial brachytherapy (IC/ISBT) for cervical cancer (CC) was modified from multiple applications $(\mathrm{MA})$ treated over 2 separate weeks $(7 \mathrm{~Gy} \times 4)$ to a single-application (SA), treated within 1 week $(8 \mathrm{~Gy} \times 3)$. Here, we assessed dosimetric quality of the SA hybrid IC/ISBT approach and report our early outcomes.

Material and methods: This was an IRB-approved retrospective review of CC patients treated with magnetic resonance imaging (MRI)-guided hybrid IC/ISBT between April 1, 2020 and December 31, 2020 (COVID cohort). Treatment parameters and quality indicators were compared to hybrid IC/ISBT cases treated in 2 years prior (pre-COVID cohort). Differences between cohorts were evaluated with the Mann-Whitney $U$-test.

Results: In the COVID compared to pre-COVID cohort, median high-risk clinical target volume (HR-CTV) was similar: 33.3 vs. $33.9 \mathrm{cc}$, as was cumulative HR-CTV $\mathrm{D}_{90 \%}: 81.2$ vs. $80.9 \mathrm{~Gy}$. Organ-at-risk $\mathrm{D}_{2 \mathrm{cc}}$ values and recto-vaginal point doses were similar. Median number of channels loaded was increased to 6 vs. $4(p=0.002)$, but percentage of total dwell time allocated to needles remained similar: $13 \%$ vs. $15 \%$. Median implant HR-CTV D $90 \%$ was higher: $107.8 \%$ vs. $98.4 \%(p=0.001)$, and there was a trend toward reduced overall treatment time (OTT): 44 days vs. 53 days $(p=0.1)$. Local control was achieved in all patients, but mucosal toxicity was higher in the COVID group, with grade 2 or higher vaginal, genitourinary, or gastrointestinal events recorded in $56 \%$ of the patients.

Conclusions: The SA hybrid IC/ISBT approach utilized during the COVID-19 pandemic maintained similar plan characteristics as pre-pandemic MA hybrid cases, while simultaneously reducing anesthesia, inpatient resources, and OTT. Local control outcomes demonstrate the regimen was effective; however, given the increased risk of mucosal toxicity, we conclude that the SA regimen should be considered only when a MA schedule is not feasible.

J Contemp Brachytherapy 2022; 14, 1: 66-71 DOI: https://doi.org/10.5114/jcb.2022.113058
\end{abstract}

Key words: cervical cancer, hybrid interstitial brachytherapy, overall treatment time, COVID-19 pandemic.

\section{Purpose}

In the early phases of the COVID-19 pandemic, at our large tertiary care academic center, both inpatient and outpatient hospital services were restructured to ensure availability of beds, personal protective equipment, testing supplies, ventilators, and trained staff. Cancer service lines were affected and physically relocated in some instances, including transfer of the inpatient women's cancer services to an off-site facility to increase intensive care capacity at the main hospital site. A 'COVID-minimal surgical pathway' was created in an effort to protect cancer patients requiring surgery from hospital-acquired COVID-19 infections [1]. This pathway mandated symptom screening, distancing, mask wearing, pre-operative COVID-19 test- ing, and a rigorous process to physically separate surgical pathway patients from the COVID-19 population. Highdose-rate (HDR) brachytherapy for cervical cancer (CC) remained an essential service with urgent tier-1 priority status within the COVID-minimal surgical pathway.

A common technique utilized at our institution for CC brachytherapy is a multiple application (MA) HDR 'hybrid' brachytherapy approach, in which interstitial brachytherapy (ISBT) needles are placed in addition to an intracavitary (IC) tandem-based applicator in the operating suite under general anesthesia (GA). For each patient, magnetic resonance imaging (MRI) with applicators in situ is obtained for image-guided 3D planning. Patients are admitted overnight for one night to the inpatient women's cancer ward in order to receive a total of 2 HDR
Address for correspondence: Shari Damast, MD, Department of Therapeutic Radiology, Yale School of Medicine, PO Box 208040, New Haven, CT 06510-8040, phone: +1 203-200-2100, fax: +1 203-785-4622, e-mail: shari.damast@yale.edu
Received: 29.07.2021

Accepted: 06.01.2022 Published: 28.01.2022 
fractions per application (7 Gy on day 1, 7 Gy the following day). The applicators are then removed, and the entire process is repeated one week later. The operational changes imposed by the pandemic presented numerous logistical challenges to this MA approach. As women's services had been moved off-site, we operationalized dedicated staffing in a non-COVID unit in the main hospital, within close proximity to the radiotherapy department. Additionally, operating suite time and GA usage needed to be minimized.

To alleviate these demands, beginning in April 2020, our institutional protocol for ISBT cases was modified to a single-application (SA) schema. The use of GA for applicator placement and MRI guidance for adaptive planning remained the same as pre-COVID; however, patients were admitted to the hospital for 2 consecutive nights, and received a total of 3 daily (8 Gy) or 5 BID (4.5-5.5 Gy) HDR fractions, using the same application. The intent was to reduce anesthesia staff/equipment involvement by half, conserve resources (PPE, staffing, testing), decrease patient visits/ potential COVID exposures, and reduce inpatient strain (bed, staffing). The chosen fractionation was the most hypo-fractionated scheme endorsed by the American Brachytherapy Society guidelines [2], and was a suggested approach in several guidelines written to lessen resource consumption in CC brachytherapy during the global pandemic [3-5].

Given the ongoing impact of COVID-19 worldwide, and the relatively limited published data examining this fractionation scheme with modern image-guided adaptive techniques, we aimed to assess the dosimetric quality of SA hybrid IC/ISBT approach and report our early outcomes.

\section{Material and methods}

We reviewed the records of stage IB3-IVA CC patients treated with MRI-guided hybrid IC/ISBT between April 1, 2020 and December 31, 2020. We excluded brachytherapy cases which were non-cervix, recurrent malignancies, and those treated with non-hybrid techniques (either IC only or ISBT). Institutional review board permission was granted for this retrospective review.

Following workup that included clinical examination, biopsy, pelvic MRI, and PET-CT scan, patients were treated with external beam radiotherapy (EBRT) to a dose of 45 Gy in 25 fractions, with concomitant weekly cisplatin chemotherapy. EBRT technique was either intensity-modulated radiotherapy (IMRT) or 3-dimensional conformal. For those with positive nodal disease, nodal boosts were delivered with an IMRT simultaneous integrated boost technique to a total dose of 55 Gy in 25 fractions. After 25 fractions of EBRT, patients were eligible for brachytherapy, which was typically administered within 1-2 weeks of EBRT completion.

For applicator placement, all patients were treated on the strict 'COVID-minimal surgical pathway', which required a negative COVID-19 test within 24 hours of surgery. As in our pre-COVID cases, all patients underwent a standardized bowel preparation beginning 24 hours prior to insertion, incorporating magnesium citrate enema and a clear liquid diet. Hybrid IC/ISBT appli- cators were placed in OR under GA, with ultrasound and fluoroscopic guidance. Patients were generally treated with an IC tandem and ring (T\&R) set, with ISBT needles placed into gross parametrial or cervical disease. Alternative applicator sets utilized included tandem and ovoid $(\mathrm{T} \& \mathrm{O})$ or tandem and cylinder (T\&C). For hybrid cases performed with T\&C, Kelowna template was used to stabilize the needles placed into residual disease involving the middle-to-lower vagina or parametrial tissues. Distribution of applicator types are included in Table 1.

All patients had MRI-based validation of applicator placement, with adjustments as necessary. MRI included T2-weighted axial, sagittal, and coronal sequences, each with 3-4 $\mathrm{mm}$ reconstructed slice thicknesses. An additional T2-weighted axial sequence aligned with the plane of the cervix was performed in all cases. MRI images were fused with a simulation CT scan for high-risk clinical target volume (HR-CTV) and organ at risk (OAR) contouring, and applicator and needle reconstruction. CT scan was performed immediately following MRI scan in the identical treatment position. Fusion followed a rigid registration algorithm with landmark points defined on the tandem and ring (or other applicable accessory, i.e., ovoids, cylinder), similar to that described by Mahantshetty et al. [6]. The MRI and CT simulation protocols were similar to those utilized pre-pandemic. Plans were developed on Eclipse BrachyVision software (version 13.6, Varian Medical Systems, a Siemens Healthineers Company), and were delivered with a GammaMed plus iX (Varian Medical Systems, a Siemens Healthineers Company) afterloader.

Patients were admitted to a special unit within a nonCOVID inpatient ward with dedicated GYN nursing and house staff, in close proximity to the radiation therapy department. Similar to our pre-COVID cases, the inpatient order set included strict bedrest precautions, limited head elevation, clear liquid diet, foley catheter to gravity, constipating medications, prophylactic blood thinning injections for deep vein thrombosis prophylaxis, patient-controlled narcotic analgesia, and around-the-clock anxiolytic medications if needed. Different from our preCOVID cases, family visitations were not permitted, but tablet computers were provided as an alternative. Patients were transported to the radiation oncology department, 1 hour prior to each planned HDR delivery for a verification simulation examination. Verification CT scans were performed prior to brachytherapy treatments on day 2 and day 3 , and were scrutinized to verify similar applicator positioning, bladder filling, and rectal emptying compared with the original planning simulation study. These image sets were registered to the CT simulation scan from day 1 , following the landmark registration algorithm described above, with alignment to the tandem and applicable accessory (i.e. ring, ovoids, cylinder). If gas was noted in the rectum, a rectal tube was employed to evacuate air if necessary, and the patient was then re-scanned. Following image registration, each needle position was then individually analyzed to confirm no displacement of the needle with respect to the applicator, HR-CTV, and OARs. Adjustments to needle position were made if needed. Each OAR in turn was individually analyzed. If a change was 
Table 1. Patient characteristics

\begin{tabular}{|c|c|c|}
\hline & $\begin{array}{c}\text { Pre-COVID } \\
\text { cohort }(n=9)\end{array}$ & $\begin{array}{l}\text { COVID cohort } \\
(n=9)\end{array}$ \\
\hline Age (years), mean (range) & $48.2(32-74)$ & $62.0(39-95)$ \\
\hline \multicolumn{3}{|l|}{ FIGO stage (2018 staging) } \\
\hline IB3 & 1 & 1 \\
\hline$\| \mathrm{A}$ & 1 & 0 \\
\hline $\mathrm{IIB}$ & 3 & 2 \\
\hline IIIA & 0 & 1 \\
\hline IIIC & 4 & 4 \\
\hline IVA & 0 & 1 \\
\hline \multicolumn{3}{|l|}{ Histology } \\
\hline Squamous & 9 & 8 \\
\hline $\begin{array}{l}\text { Adeno or } \\
\text { adenosquamous }\end{array}$ & 0 & 1 \\
\hline $\begin{array}{l}\text { PET pre-Tx (SUVm), } \\
\text { median (range) }\end{array}$ & $12.6(8.1-25.9)$ & $15.1(7.1-22)$ \\
\hline \multicolumn{3}{|l|}{$\begin{array}{l}\text { Hybrid implant dose } \\
\text { schema (cGy) }\end{array}$} \\
\hline MA $700 \times 2$ repeat $\times 2$ & 7 & 0 \\
\hline SA $450 \times 5$ BID & 0 & 1 \\
\hline SA $500 \times 5$ BID & 1 & 0 \\
\hline SA $550 \times 5$ BID & 0 & 2 \\
\hline SA $800 \times 3$ daily & 1 & 6 \\
\hline \multicolumn{3}{|l|}{ Applicator type } \\
\hline$T \& C+I S B T$ & 1 & 2 \\
\hline$T \& O+I S B T$ & 0 & 1 \\
\hline$T \& R+I S B T$ & 8 & 6 \\
\hline $\begin{array}{l}\text { Number of channels, } \\
\text { median (range) }\end{array}$ & $4(4-7)$ & $6(3-10)$ \\
\hline \multicolumn{3}{|l|}{$\begin{array}{l}\text { Ratio of dwell times/ } \\
\text { channel to total implant } \\
\text { dwell time (median \%) }\end{array}$} \\
\hline Tandem & 65 & 64 \\
\hline Ov/ring/cyl & 20 & 23 \\
\hline Needles & 15 & 13 \\
\hline $\begin{array}{l}\text { HR-CTV volume (cc), } \\
\text { median (range) }\end{array}$ & $33.9(19.0-61.0)$ & $33.3(24.8-53.3)$ \\
\hline
\end{tabular}

\begin{tabular}{lcc} 
& $\begin{array}{c}\text { Pre-COVID } \\
\text { cohort }(n=9)\end{array}$ & $\begin{array}{c}\text { COVID cohort } \\
(n=9)\end{array}$ \\
\hline $\begin{array}{l}\text { HR-CTV } \mathrm{D}_{90 \%} \text { (\% of } \\
\text { brachytherapy prescription } \\
\text { dose), median (range) }\end{array}$ & $98.4(80.6-104.6)$ & $107.8(90.6-116.7)$ \\
\hline $\begin{array}{l}\text { Cumulative HR-CTV } \mathrm{D}_{90 \%,} \\
\text { including EBRT (Gy, EQD }), \\
\text { median (range) }\end{array}$ & $80.9(70.5-88.2)$ & $81.2(75.6-88.8)$ \\
\hline $\begin{array}{l}\text { Volume of HR-CTV re- } \\
\text { ceiving at least 200\% of }\end{array}$ & & \\
prescribed dose $\left(\mathrm{V}_{200 \%}\right)$ & & \\
\hline Median in cc (range) & $11.5(6.0-21.8)$ & $12.1(7.0-16.2)$ \\
\hline Median in \% (range) & $31.5(25.2-42.4)$ & $34.1(28.1-43)$ \\
\hline $\left.\begin{array}{l}\text { Median } \mathrm{D}_{2 c c} \text { OARs doses, } \\
\text { including EBRT (Gy, EQD }\end{array}\right)$ & & \\
\hline Bladder & $75.5(66.1-82.7)$ & $74.2(56.2-81.8)$ \\
\hline Rectum & $51.4(48.5-68.5)$ & $52.6(48.4-67.7)$ \\
\hline Sigmoid & $62.0(46.1-66.7)$ & $55.1(47.9-61.6)$ \\
\hline Bowel & $56.7(47.8-74.2)$ & $58.7(56.4-69.6)$ \\
\hline
\end{tabular}

Cumulative vaginal dose

points (Gy, EQD 2 )

\begin{tabular}{ccc}
\hline Max vaginal point & $122.4(118.2-133.5)$ & $106.5(91.0-125.0)$ \\
\hline Recto-vaginal point & $53.4(51.3-57.6)$ & $51.6(47.1-71.3)$ \\
\hline OTT, median (range) & $53(43.0-62.0)$ & $44(42.0-65.0)$ \\
\hline
\end{tabular}

Follow-up time (months), $\quad 24.9$ (16.1-47.4) 15.7 (14-22.9)

median (range)

\begin{tabular}{lcc}
\hline Complete local response & $100.0 \%$ & $100.0 \%$ \\
\hline $\begin{array}{l}\text { PET post-Tx (SUVm), } \\
\text { median (range) }\end{array}$ & $3.1(0.0-5.4)$ & $3.0(0.0-7.2)$ \\
\hline
\end{tabular}

median (range)

\begin{tabular}{lll}
\hline Grade 2-3 complications & & \\
\hline Vesico-vaginal fistula & 0 & 1 \\
\hline Hemorrhagic cystitis & 0 & 1 \\
\hline Rectovaginal fistula & 0 & 1 \\
\hline Cervical necrosis & 0 & 1 \\
\hline Vaginal stenosis & 0 & 1 \\
\hline Local recurrence & 0 & 0 \\
\hline Distant recurrence & 3 & 1
\end{tabular}

FIGO - International Federation of Gynecology and Obstetrics; PET - positron emission tomography; SUV - standardized uptake value; MA - multiple applications; $S A$ - single application; T\&C - tandem and cylinder; ISBT - interstitial brachytherapy; T\&O - tandem and ovoid; T\&R - tandem and ring; HR-CTV-high-risk clinical target volume; OARs - organs at risk; EQD2 - equivalent dose in $2 \mathrm{~Gy} /$ fraction calculated assuming $\alpha / \beta=10$ Gy for HR-CTV or 3 Gy for OARs; EBRT - external beam radiotherapy; OTT - overall treatment time

noted in the position of OAR with respect to the implant, the OAR was re-contoured so that max point and $\mathrm{D}_{2 \mathrm{cc}}$ values could be re-calculated. Re-planning was not done routinely; however, it was available if there was significant variability in OAR dose with respect to the original plan. For patients who were treated with BID fractionation, CT simulation scan was used only prior to the morning delivery. Prior to the afternoon fractionation, fluoroscopic c-arm imaging in the brachytherapy suite was used to confirm consistent geometry of the needles with respect to intra-cavitary applicator.
In general, the goal $\mathrm{EQD}_{2}$ with cervical HDR brachytherapy is $\sim 40$ Gy in LDR equivalent. Assuming $\mathrm{a} / \beta=10 \mathrm{~Gy}$, this threshold is reached with common regimens, i.e., $7 \mathrm{~Gy} \times 4$ or $6 \mathrm{~Gy} \times 5$. However, a SA approach of $8 \mathrm{~Gy} \times 3$ provides only $36 \mathrm{~Gy} \mathrm{EQD}_{2}$. To compensate for the lower $\mathrm{EQD}_{2}$, an a priori decision was made to produce a globally 'hotter' implant to maintain GEC-ESTRO and ABS guidelines [2]. We relaxed our implant HR-CTV $\mathrm{D}_{90 \%}$ coverage upper limit from $110 \%$ to $117 \%$ (resultant increase in cumulative dose from 85.6 Gy EQD 2 to $89.6 \mathrm{~Gy} \mathrm{EQD}_{2}$ when combined with the 
45 Gy EBRT dose), to reflect published trends suggesting improved outcomes with doses up to $90 \mathrm{~Gy} \mathrm{EQD}$. For cases in which HR-CTV included substantial portions of the middle and lower vagina, the goal cumulative HR-CTV D90\% was lower (around 75 Gy EQD 2 ). Despite the 'hotter' implant allowance, our clinical goals continued from our pre-pandemic cases, maintaining a total interstitial needle component $<15 \%$ of the overall dwell time (i.e., $85-90 \%$ overall dwell time in IC channels).

OAR constraints remained unchanged from prepandemic, with $\mathrm{D}_{2 \mathrm{cc}}$ aims (limits), in units of $\mathrm{EQD}_{2}$ : bladder $\leq 80$ (85), rectum $\leq 65$ (75), sigmoid $\leq 70$ (75), and bowel $\leq 65$ (75). EQD 2 for OARs was calculated assuming $\alpha / \beta=3$ Gy. The ICRU recto-vaginal $(R-V)$ dose point was retrospectively analyzed and compared between the two groups. Additionally, at our institution, we report an upper vaginal mucosa maximum point dose defined at the outer surface of the ring or ovoid on the left and right side, with an aim of 120 Gy (limit, 140 Gy).

We compared clinical characteristics and dosimetric parameters of the COVID patients treated ('COVID cohort') to those of consecutive MRI-guided hybrid ISBT cases treated in the 2 years prior ('pre-COVID cohort'). Dosimetric parameters included number of channels loaded per implant, HR-CTV volume, HR-CTV $\mathrm{D}_{90 \%}$, OAR $\mathrm{D}_{2 \mathrm{cc}}$ maximum vaginal dose points, $\mathrm{R}-\mathrm{V}$ dose point, $\mathrm{V}_{200 \%}$, and overall treatment time (OTT) from start of external beam to completion of brachytherapy. Descriptive statistics were performed in Excel; differences between cohorts were evaluated with Mann-Whitney $U$-test. Descriptive outcomes included response rates, disease progression, and rate of grade 2 or higher toxicities (according to the common terminology for clinical adverse events, version 4.0).

\section{Results}

Characteristics of both the COVID and pre-COVID cohorts are shown in Table 1.

In the COVID cohort $(n=9)$, the patients were prescribed SA 8 Gy $\times 3(n=6)$ or 4.5-5.5 Gy $\times 5$ BID $(n=3)$ delivered over 3 consecutive days. In the pre-COVID cohort $(n=9)$, the most common hybrid prescription was 7 Gy $\times 4(n=7)$ with MA (first application with implants delivered on days 1, 2; second application with implants on days 8,9$)$, whereas SA prescriptions included $5 \mathrm{~Gy} \times$ 5 BID $(n=1)$ and 8 Gy $\times 3(n=1)$. Fraction of $7-8$ Gy were separated by a minimum of 18 hours; fraction sizes of 4.5-5.5 Gy were separated by a minimum of 6 hours.

The median number of channels loaded was increased in the COVID cohort to 6 (range, 3-10) versus 4 in the preCOVID cohort (range, 4-7) $(p=0.003)$. Increased number of needles were used in the SA COVID cohort for two reasons: 1 . To provide generous geographic coverage of HR-CTV, since there would not be a second implant to make up for potential cold spots as there would be with an MA approach; and 2. To provide generous dosimetric coverage of HR-CTV to reasonably achieve higher HR-CTV $\mathrm{D}_{90 \%}$ coverage goal despite the inherently lower $\mathrm{EQD}_{2}$ associated with SA. The median implant HR-CTV $\mathrm{D}_{90 \%}$ for the COVID cohort was higher: $107.8 \%$ (90.6$116.7 \%)$ vs. $98.4 \%(80.6-104.6 \%)(p=0.001)$ (calculated as percentage dose, for brachytherapy portion of treatment only). However, the $\mathrm{V}_{200 \%}$ values were similar: $34.1 \%$ vs. $31.5 \%$. The ratio of ISBT-to-total dwell time were similar: $13 \%$ vs. $15 \%$. An illustration of an IC/ISBT case of R\&T with 6 interstitial needles ( 4 titanium and 2 PEEK) is presented in Figure 1.

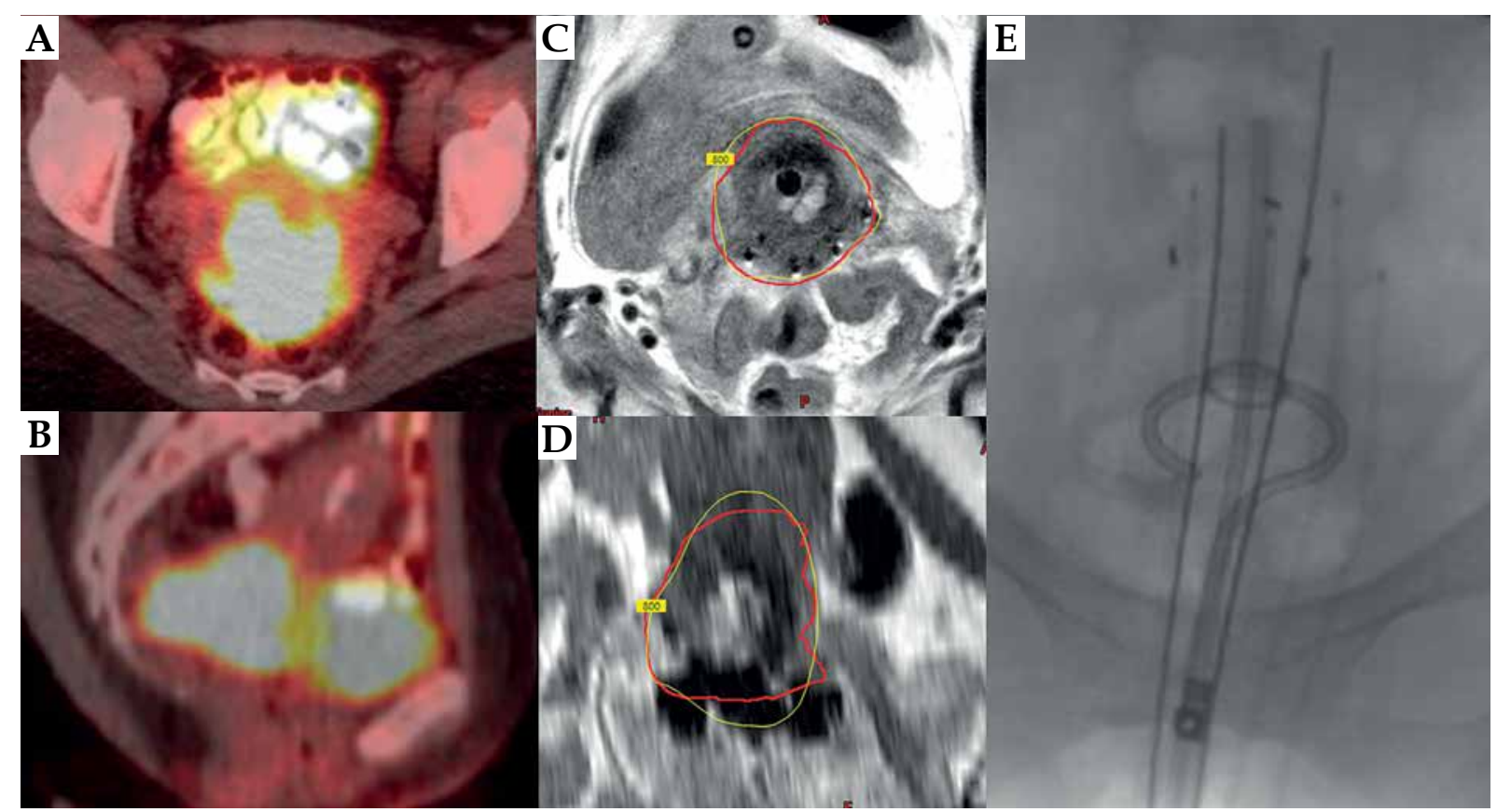

Fig. 1. MRI-guided hybrid interstitial brachytherapy for a patient with stage IIB SCC. A, B) PET imaging at diagnosis. C, D) Hybrid IC/ISBT implant. The red contour is the HR-CTV. The yellow isodose line is the prescription of $800 \mathrm{cGy}$. E) A radiograph displaying the relationship of needles to the tandem and ring. In this case, there were 4 needles inserted through a Vienna ring cap parallel to the tandem and 2 additional oblique needles 
In terms of dosimetry, as shown in Table 1, the median HR-CTV volume was similar: $33.3 \mathrm{cc}$ vs. $33.9 \mathrm{cc}$ (range, 24.8-53.3 cc and 19-61 cc for COVID and pre-COVID cohorts, respectively), as was cumulative HR-CTV $\mathrm{D}_{90 \%}$ including dose contribution from EBRT: 81.2 vs. 80.9 Gy $\mathrm{EQD}_{2}$. The bladder, rectum, sigmoid, and bowel $\mathrm{D}_{2 \mathrm{cc}}$ values were also similar. The R-V dose points were similar between the cohorts; however, the maximum vaginal dose point in $\mathrm{EQD}_{2}$ was higher in the pre-COVID cohort $(p=0.003)$. Importantly, there was a trend toward reduced OTT by greater than 1 week in the COVID compared to pre-COVID groups: 44 days (range, 42-65) vs. 53 days (range, 43-62) ( $p=0.1$ ).

All the patients in both cohorts have had complete local response. The median follow-up time is longer in the pre-COVID (24.9 months, range: 16.1-47.4 months) compared to COVID group (15.7 months, range: 14-22.9 months). In the pre-COVID cohort, there were 3 cases of distant recurrences, whereas, thus far, there has been only 1 distant recurrence in the COVID group. There were no grade 3 or higher toxicities in the pre-COVID cohort. In the COVID cohort, there were 2 grade 3 genitourinary toxicities, 1 grade 2 rectal toxicity, and 2 grade 3 vaginal toxicities, for a total of 5 grade 2 or higher events. The two grade 3 genitourinary toxicities included one patient (stage IVA, bladder involvement, bladder $\mathrm{D}_{2 \mathrm{cc}}=$ $81.8 \mathrm{~Gy}$ ) who developed a vesicovaginal fistula 6 months post brachytherapy and one patient (stage IIB, bladder $\left.\mathrm{D}_{2 \mathrm{cc}}=74.2 \mathrm{~Gy}\right)$ who developed grade 3 hemorrhagic radiation cystitis 13 months post brachytherapy. Notably, the patient with a fistula was a heavy smoker and malnourished (on total parenteral nutrition), and these were felt to be risk factors for fistula in addition to substantial bladder involvement at the time of diagnosis. One patient (stage IIIC1, rectal $\mathrm{D}_{2 \mathrm{cc}}=62 \mathrm{~Gy}$ ) developed a small rectovaginal fistula (grade 2 , conservative non-invasive management) 11.5 months post brachytherapy. Of note, she had been taking axitinib for an unrelated diagnosis of adenoid cystic carcinoma of the salivary gland, a drug which has been associated with risk of intestinal fistula. Additionally, there were two patients who developed grade 3 vaginal or cervical mucosal complications. One patient with stage IIIA disease with lower $1 / 3$ vaginal involvement developed grade 3 vaginal stenosis at 4 months post-brachytherapy. Her implant HR-CTV $\mathrm{D}_{90 \%}$ was $114 \%$ of the prescription dose, and her OTT was 44 days. Additional risk factors for vaginal stenosis were due to the extent of her disease, and the necessity to treat the entire length of the vaginal canal to a minimum dose of $65 \mathrm{~Gy} \mathrm{EQD} \mathrm{E}_{2}$, resulting in $\mathrm{R}-\mathrm{V}$ point max $>$ 65 Gy. Further, she was not compliant with vaginal dilator therapy post-treatment. Though the patient remains without evidence of disease or symptoms, this was considered a grade 3 vaginal mucosal toxicity per CTCAE version 4.0 criteria due to the inability to perform an adequate physical examination. One patient with stage IB3 disease developed cervical necrosis at 5 months after brachytherapy. Of note, she had high implant HR-CTV $\mathrm{D}_{90 \%}$ coverage (115\%, total cumulative $\mathrm{EQD}_{2} 88.2 \mathrm{~Gy}$ ) and short OTT (42 days). The extent of her necrosis prompted a surgical workup, which consisted of biopsies and surgical debridement.

\section{Discussion}

The COVID-19 pandemic necessitated operational changes across hospitals, cancer centers, and radiation facilities worldwide. Various evidence-based algorithms were disseminated to promote a rational framework for triaging cancer services. Radiotherapy for CC remained a tier-1 life-saving treatment with provision of high quality services, such as brachytherapy known to preserve life. International expert recommendations supported the use of $8 \mathrm{~Gy} \times 3$ or other hypo-fractionated regimens to maximize efficiency, conserve resources, and reduce risk for potential COVID-19 transmission [3-5]. We have shown high rates of local control and cure for CC patients despite the pandemic, and the required modifications throughout our health system, which necessitated BT delivery in an efficient, less resource-intense manner using a SA hybrid IC/ISBT approach. However, the higher rate of mucosal toxicity observed compared to our pre-pandemic cases was notable and deserves further discussion.

A common dose schema used internationally for hybrid brachytherapy for CC is 7 Gy $\times 4$, with MA, over 2 separate weeks [7]. There are other acceptable schema based on experiences from the US and worldwide [2]. The 8 Gy $\times 3$ fractionation is supported by a randomized, controlled trial from India that showed equal local control and toxicity compared to a 4-fraction regimen, while simultaneously increasing acceptability to patients and compliance due to shorter schedule and fewer fractions [8]. Our regimen of $8 \mathrm{~Gy} \times 3$ however differed from this trial in two major ways. First, our patients were treated daily over 3 days with a SA, whereas in the trial, patients were treated with MA, once weekly, for 3 weekly treatments. Second, all of our patients in this series received hybrid IC/ISBT, whereas the trial from India utilized IC applicators only. The hybrid technique allowed substantial sparing of the surrounding OARs, with simultaneous dose-escalation to the target HR-CTV. The SA hybrid approach used in our study was similar to that used by Mahantshetty et al. in their recent experience of 38 patients, who were treated with a single-application, multi-fractionated regimen in India [6]. Following a dose of $50 \mathrm{~Gy}$ to the pelvis, the authors prescribed a regimen of 3 fractions of SA with 9 Gy on day 1, and two fractions of 7 Gy delivered BID on day 2, separated by at least 6 hours. All patients were treated with a hybrid IC/ISBT approach, with 4 needles used on average. Mean OTT was 47 days and their 2-year local control was $90 \%$.

Overall, similar to the findings of Mahntshetty et al., we found that cancer outcomes were excellent with no local recurrences. We found that the SA rather than MA approach was associated with a shorter OTT of 44 days. We hypothesize that the shorter OTT may have had a positive effect on recurrence-free survival, reflecting the recently published EMBRACE nomogram, in which shorter OTT corresponded with better outcomes [9]. However, it may also be possible that the shorter OTT contributed to the high rate of mucosal toxicity (56\% grade $2-3$ in the 
COVID cohort), in combination with the hotter implants and/or the contracted schedule of delivery over a single application. In the Mahantshetty trial, two grade 3 rectal toxicities were recorded. The authors did not report on cervical or vaginal mucosal complications, and concluded that longer follow-up is needed to evaluate late toxicities to surrounding organs. Whether equally high dose is needed to treat CC when the OTT is shortened deliberately may be a topic deserving future prospective investigation.

To make up for the lower $\mathrm{EQD}_{2}$ with the SA fractionation, and in an effort to achieve the modern recommended cumulative HR-CTV $\mathrm{D}_{90 \%}$ goal of 85-90 Gy (including EBRT), we had added more needles to produce a 'hotter' implant, which resulted in a median implant HR-CTV $\mathrm{D}_{90 \%}$ dose of approximately $10 \%$ hotter than that of our pre-COVID cases. In some cases, with $\mathrm{D}_{90 \%}$ limit relaxed to $115-117 \%$ prescription dose, the implant HR-CTV $\mathrm{D}_{90 \%}$ received nearly 9.2-9.4 Gy (physical dose)/fraction. However, in retrospect, this may have resulted in unanticipated high biologic doses to normal tissues, including the vagina and uninvolved cervix. While the dose to OARs including the vagina were all within acceptable limits, and metrics including percent loading of needles and $\mathrm{V}_{200 \%}$ were similar to the MA regimen, the biologic impact of high-dose per fraction and the accelerated time of treatment may not be accounted for in standard $\mathrm{EQD}_{2}$ dose calculations. Based on this experience, our preference is to treat patients with a MA regimen when feasible, and a fraction size no larger than 7 Gy. However, if SA is the only viable method of treatment for a given patient, then we restrict the implant HR-CTV $D_{90 \%}$ to $<110 \%$ of the brachytherapy prescription dose as an upper limit, and enforce the stricter lower boundaries of published bladder, rectal, or bowel constraints.

There are several limitations to our study. First, this work represents the findings from a solution implemented at a single institution in response to an unprecedented crisis, during a time in which bringing a patient into the hospital also posed grave threat to patients' health and mortality from COVID-19. Secondly, the cohort is small and follow-up is not mature. Thus, it is challenging to draw firm conclusions nor can they be generalized. $8 \mathrm{~Gy} \times 3$ is not an uncommon dose/fractionation schema in practice and it is endorsed by societal guidelines [2-5]. Treatment with a weekly rather than daily frequency may not carry the same risk of toxicity that were seen with the SA approach used in this study.

\section{Conclusions}

The SA IC/ISBT approach utilized during the COVID-19 pandemic achieved dosimetric quality measures comparable to our pre-pandemic MA cases, while reducing anesthesia, PPE, and inpatient resources. An additional advantage included shorter OTT. Local control outcomes demonstrate the regimen was effective for CC; however, going forvard, given the increased risk of mucosal toxicity observed, we conclude that the SA regimen should be used with caution and only considered in cases for which a MA schedule is not a feasible alternative.

\section{Disclosure}

The authors report no conflict of interest.

\section{References}

1. Boffa DJ, Judson BL, Billingsley KG et al. Results of COVID-minimal surgical pathway during surge-phase of COVID-19 pandemic. Ann Surg 2020; 272: e316-320.

2. Albuquerque K, Hrycushko BA, Harkenrider MM et al. Compendium of fractionation choices for gynecologic HDR brachytherapy - an American Brachytherapy Society Task Group Report. Brachytherapy 2019; 18: 429-436.

3. Mendez LC, Raziee H, Davidson M et al. Should we embrace hypofractionated radiotherapy for cervical cancer? A technical note on management during the COVID-19 pandemic. Radiother Oncol 2020; 148: 270-273.

4. Elledge CR, Beriwal S, Chargari C et al. Radiation therapy for gynecologic malignancies during the COVID-19 pandemic: International expert consensus recommendations. Gynecol Oncol 2020; 158: 244-253.

5. Miriyala R, Mahantshetty U. Brachytherapy in cervical cancer radiotherapy during COVID-19 pandemic crisis: problems and prospects. J Contemp Brachytherapy 2020; 12: 290-293.

6. Mahantshetty U, Gurram L, Bushra S et al. Single application multi-fractionated image guided adaptive high dose rate brachytherapy for cervical cancer - dosimetric \& clinical outcomes. Int J Radiat Oncol Biol Phys 2021; 111: 826-834.

7. Pötter R, Tanderup K, Schmid MP et al. EMBRACE Collaborative Group. MRI-guided adaptive brachytherapy in locally advanced cervical cancer (EMBRACE-I): a multicentre prospective cohort study. Lancet Oncol 2021; 22: 538-547.

8. Rao BS, Das P, Subramanian BV et al. A Comparative analysis of two different dose fractionation regimens of high dose rate intracavitary brachytherapy in treatment of carcinoma of uterine cervix: a prospective randomized study. J Clin Diagn Res 2017; 11: XC06-10.

9. Sturdza AE, Poetter R, Kossmeier M et al. Nomogram predicting overall survival in locally advanced cervical cancer patients treated by radio-chemotherapy including image guided brachytherapy: a Retro-EMBRACE study. Int J Radiat Oncol Biol Phys 2021; 111: 168-177. 